\title{
Comparison of different pedicle screw fixation schemes in the treatment of neurosurgical spinal fractures: systematic review and meta-analysis
}

\author{
Dan Liang ${ }^{1}$, Xiaopeng Deng ${ }^{1}$, Jiacai Qian ${ }^{1}$, Feng Han ${ }^{2}$, Kun Zhou ${ }^{1}$ \\ ${ }^{1}$ Neurosurgery Department, The Second People's Hospital of Guiyang, Guiyang, China; ${ }^{2}$ Neurosurgery Department, The Affiliated Hospital of \\ Guizhou Medical University, Guiyang, China \\ Contributions: (I) Conception and design: D Liang, K Zhou; (II) Administrative support: X Deng; (III) Provision of study materials or patients: X \\ Deng, J Qian, F Han, K Zhou; (IV) Collection and assembly of data: All authors; (V) Data analysis and interpretation: D Liang, J Qian, F Han, K \\ Zhou; (VI) Manuscript writing: All authors; (VII) Final approval of manuscript: All authors. \\ Correspondence to: Kun Zhou. Neurosurgery Department, The Second People's Hospital of Guiyang, No. 547, Jinyang South Road, Guanshanhu \\ District, Guiyang, China. Email: zkk18908506555@163.com.
}

Background: Thoracolumbar fractures have the characteristics of acute onset, rapid change, and severe
trauma. The best way to treat thoracolumbar fractures is through fracture reduction surgery. The surgical
methods include percutaneous pedicle screw, posterior percutaneous pedicle screw internal fixation, and
open pedicle screw internal fixation. Methods: We searched the PubMed, Embase, and Medline English database from April 1991 to April 2021, and the keywords included "percutaneous tablet screen", "posterior percutaneous tablet screen fixation", "open tablet screen fixation", "fracture of thoracic vertebrae", "thoracic fractures", "thoracic", and "vascular fracture". RevMan5.3 provided by Cochrane was used for meta-analysis.

Results: A total of 9 articles were included in this study. Percutaneous pedicle screw fixation and posterior percutaneous pedicle screw fixation were adopted as the surgical methods, and patients were enrolled into experimental and control groups. Open pedicle screw internal fixation was set as the control group. The mean difference (MD) of operation time, blood loss, pain score, postoperative complications, screw debris rate, and hospital stay were $-0.73,-192.16,-0.70,1.49,0.32$, and -1.26 , respectively; $95 \%$ confidence intervals (CIs) were $(-0.94,-0.51),(-213.23,-171.09),(-0.82,-0.57),(0.47,4.79),(0.10,0.99)$, and $(-1.82$, $-0.71)$, respectively; $Z$ values were $6.71,17.87,10.95,0.67,1.97$, and 4.46 , respectively; and $\mathrm{P}$ values were $<0.00001,<0.00001,<0.00001,0.50,0.05$, and $<0.00001$, respectively.

Discussion: A total of 9 articles were included in this meta-analysis. Compared with open surgery, the use of percutaneous pedicle screw and posterior percutaneous pedicle screw fixation had less blood loss, shorter operation time, shorter hospital stay, less pain, as well as lower screw dislocation and postoperative infection rates, indicating that the use of percutaneous pedicle screw and posterior percutaneous pedicle screw fixation is more effective than open surgery.

Keywords: Percutaneous pedicle screw; posterior percutaneous pedicle screw internal fixation; open pedicle screw internal fixation; meta-analysis

Submitted Oct 31, 2021. Accepted for publication Dec 16, 2021.

doi: 10.21037/apm-21-3533

View this article at: https://dx.doi.org/10.21037/apm-21-3533 


\section{Introduction}

Spinal fracture is a common condition, among which thoracic fracture has the highest incidence (1). It has attracted extensive attention because of its potential to affect the central nervous system. Severe vertebral fractures, if not treated promptly and thoroughly, can result in spinal cord injury, then leading to more serious complications $(2,3)$. Spinal fracture is a significant harm to the patient and a huge burden to the patient's family after paralysis. Therefore, to realize optimal recovery and minimize the time and cost of surgery, the effective surgical treatment of thoracic vertebral fracture was explored in this work. In 1983, Denis (4) proposed a new classification method according to hundreds of experiences treating of patients with spinal injury, in which the thoracolumbar spine was classified into anterior, middle, and posterior columns. The anterior column referred to the anterior longitudinal ligament, the anterior half of the vertebral body, and the anterior part of the intervertebral disc. The mid-column included the posterior longitudinal ligament, the posterior half of the vertebral body, and the posterior part of the intervertebral disc. The posterior column included the vertebral arch, ligamentum flavum, intervertebral facet joint, and interspinous ligament.

Later researchers refined Denis' theory of the three columns by suggesting that the vertebral body and posterior third of the interdict should be placed in the column of the three-column classification of the spine (5). All spinal fractures with injuries to the stele should be included in the classification of spinal fractures as unstable. Based on the French Roy-Camille classification, the articular process and pedicle should also be included in the middle column of the three-column classification of the spine. However, they maintained that all spinal fractures with injuries to the stele should be included in the classification of spinal fractures as unstable (6). Currently, the opening of the open vertebral screw fixation is widely used in clinical application, which can immediately restore the normal sequence of spine and maintain the stability of the spine (7). Researchers such as Keorochana et al. [2017] (8) were comparative analysis of pedicle screw and cortical bone trajectory (CBT) for posterior lumbar interbody fusion (PLIF) postoperative fixing effect, and it is pointed out that the CBT is easier to trigger after PLIF. There is the occurrence of complications such as leg pain. In addition, some researchers pointed out that open surgery can cause severe trauma to patients with vertebral root screw incision, long operation time, large intraoperative blood volume, long-lasting incision pain in patients after surgery, and general postoperative renewal after surgery. The drainage tube leads to the prolong of the patient's bed, hospitalization, and recovery time, accompanied by stiffness, pain, and concurrent symptoms (9). How to reduce and alleviate the medical source caused by the postoperative screw fixation operation still needs to be explored.

As minimally invasive concepts are in-depth clinical, post-transparent vertebrae screws are widely used in the treatment of thoracic fractures. The blood vertebrae screw and the rear pathogenesis were placed in the subcutaneous fixation, which not only retained the advantages of satisfaction of open surgery, but also fixed strongly and reduced the damage to the back of the lumbar vertebrae. It has the advantages of simple operation, small trauma, less bleeding, short operation time, and fast postoperative recovery. The clinical effect is ideal (10). Moreover, researchers pointed out that the surgical time and radiation in this surgical method will have an effect of treatment (11). It is concluded that there is currently controversial in the treatment effect of neurosurgical spinal fractures for different pedicle screw fixation. The existing reports were systematically analyzed in this work, to provide comprehensive quantitative indicators and evidence based on post-clinical treatment.

We present the following article in accordance with the PRISMA reporting checklist (available at https://dx.doi. org/10.21037/apm-21-3533).

\section{Methods}

\section{Literature search strategy}

We searched the PubMed, Embase, and Medline English database from period between April 1991 and April 2021. "Percutaneous pedicle screw", "posterior percutaneous pedicle screw fixation", "Open pedicle screw fixation", "fracture of thoracic vertebrae", "thoracic fractures", "thoracic vertebral fracture", etc. were set as the search terms. The literature was then screened according to inclusion and exclusion criteria.

\section{Inclusion and exclusion criteria}

Articles were included in this meta-analysis if they met the following inclusion criteria: (I) recombination of experimental data with required basic information; (II) the 
disease was thoracolumbar vertebral fracture; (III) adequate number of cases with a sufficiently large sample; and (IV) clinical trials.

Articles were excluded from the meta-analysis if they met any of the following exclusion criteria: (I) non-English literature; (II) other meta-analyses; (III) non-clinical trials; (IV) unpublished articles; (V) studies with no control group; (VI) incomplete basic data records of patients.

\section{Data extraction}

The initial outcome measures included surgical parameters, postoperative pain, postoperative complications, and postoperative imaging parameters. Surgical parameters included duration of surgery, intraoperative blood loss, and length of hospital stay with minimally invasive percutaneous pedicle screws versus traditional open pedicle screws. Postoperative pain was evaluated using the visual analogue scale (VAS) between minimally invasive percutaneous pedicle screws and traditional open pedicle screws. The postoperative complications of minimally invasive percutaneous pedicle screws and traditional open pedicle screws mainly included the dislocation rate and infection rate of the postoperative screws. Image parameters included the surgical segment Cobb angle after minimally invasive percutaneous pedicle screws and traditional open pedicle screws, the postoperative Cobb angle of the fractured vertebra itself, and the postoperative vertebral anterior edge height. For studies that did not report accurate means and standard deviations (SDs), estimates were made using the graphs used in the literature. All data were extracted from the included articles. The literature was independently reviewed, and the quality of each article was assessed by two reviewers. Disagreements between the reviewers were resolved through discussion and negotiation or third-party reviewer intervention.

\section{Quality assessment}

The quality of the reviewed literature was assessed in accordance with the "risk of bias assessment" recommended in version 5.3 of the Cochrane system review manual. The evaluation included the following seven items: (I) which random method was used; (II) whether allocation concealment was used; (III) whether the blind method was used for patients and researchers; (IV) the effectiveness of the blinding method; (V) whether the research results were clear and definite; (VI) whether the intention processing analysis method was used to process the results; and (VII) other biases. Satisfying with the criterion means a small bias, and dissatisfying means a high bias. If not mentioned, the risk includes four dimensions of random sequence generation, allocation concealment, blind method, and tracking/exit, with a score of 1 to 3 as low quality and a score of 4 to 7 as high quality.

\section{Statistical processing}

RevMan5.3.5 provided by the Cochrane collaboration was used to conduct data analysis. Dichotomous variables were evaluated by the relative risk (RR) and odds ratio (OR), while the weighted mean difference (WMD) was used to evaluate the continuous variables, whose $95 \%$ confidence intervals (CIs) were calculated. As for the heterogeneity of literature, the $\chi^{2}$ test was mainly used to investigate the homogeneity of the included studies. If $\mathrm{P}>0.1$ and $\mathrm{I}^{2}<50 \%$, the included literatures were considered homogenous or consistent, and the fixed effect model was used. However, if $\mathrm{P}<0.1$ and $\mathrm{I}^{2}>50 \%$, it was considered that there is heterogeneity in the included literature data, and the random effects model was used for analysis. The test level of the meta-analysis was set as $\alpha=0.05$, and $P \leq 0.05$ was considered statistically significant.

\section{Results}

\section{Literature search results}

In this work, 570 articles were retrieved from the PubMed database, 446 articles were retrieved from the Embase database, and 258 articles were retrieved from the Medline database. After the titles and abstracts were reviewed, 623 literatures that clearly did not meet the inclusion criteria were excluded. Then, five more articles were excluded after review of the full texts, and 9 literatures (12-20) that met the inclusion criteria were finally included (Figure 1). In the inclusion in the literature, the study of minimally invasive transparent vertebrae screws was used, including two postgraduated vertebrae root screws, and the traditional open vertebra roots are used in 9 articles. Screws were fixed as a control (Table 1).

\section{Bias-risk assessment of included articles}

The Cochrane Handbook (version 5.0.2) systematic review writing manual was used to evaluate the risk of bias in the 


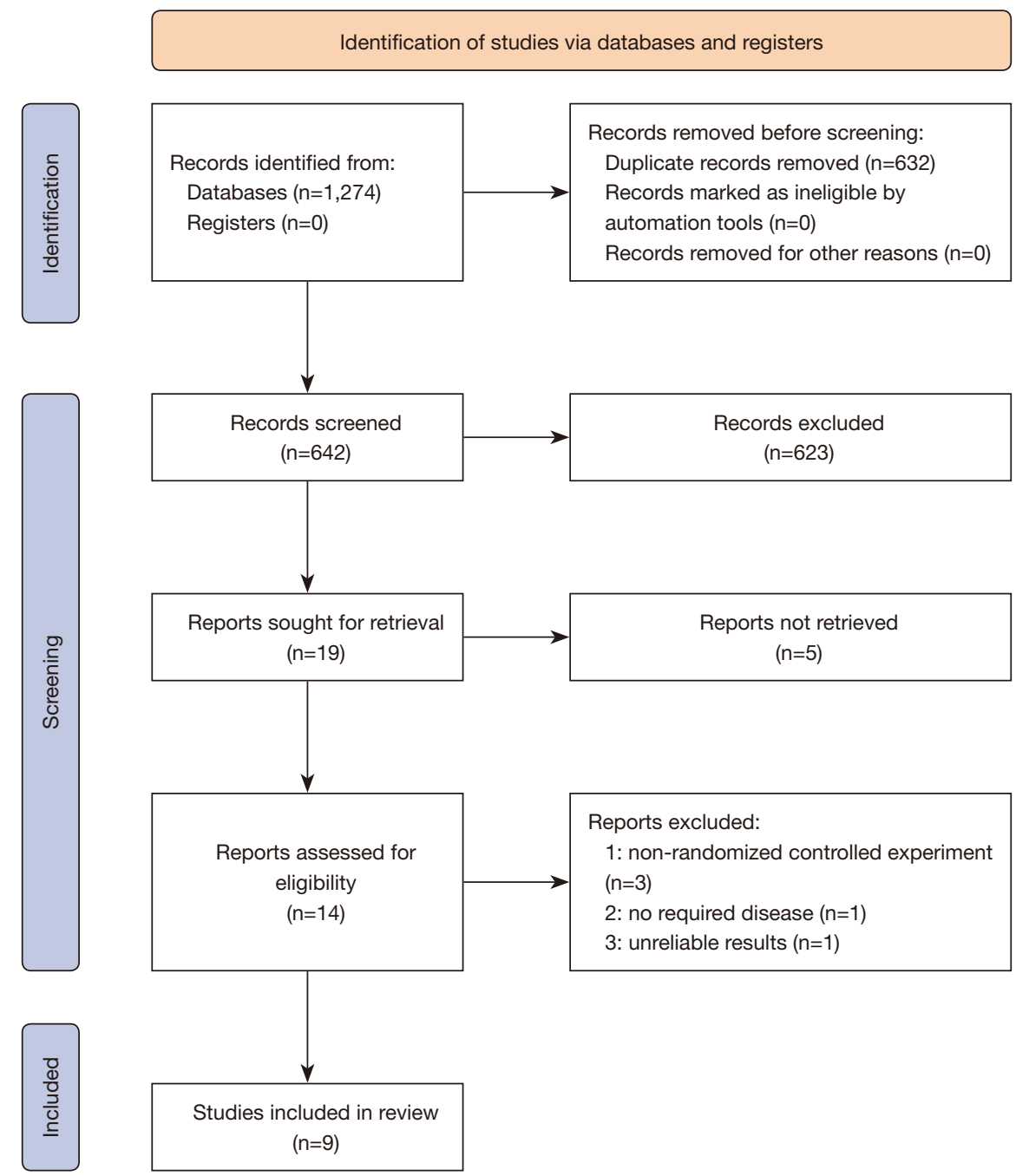

Figure 1 Literature retrieval process.

Table 1 Basic characteristics of the included literature

\begin{tabular}{|c|c|c|c|c|c|}
\hline First author & Year & The research type & Operation method [example] & $\begin{array}{l}\text { Open surgery } \\
\text { (example) }\end{array}$ & $\begin{array}{l}\text { Follow-up time } \\
\text { (months) }\end{array}$ \\
\hline Bronsard (12) & 2013 & Observational study & Minimally invasive percutaneous surgery [30] & 30 & 25.5 \\
\hline Grass (13) & 2006 & Observational study & Minimally invasive percutaneous surgery [33] & 24 & Not mentioned \\
\hline Grossbach (14) & 2013 & Observational study & Minimally invasive percutaneous surgery [11] & 27 & 18.5 \\
\hline Lyu (17) & 2016 & Randomized controlled study & Posterior percutaneous surgery [60] & 60 & 12 \\
\hline Vanek (18) & 2014 & Observational study & Minimally invasive percutaneous surgery [18] & 19 & 24 \\
\hline Wang (19) & 2017 & Observational study & Minimally invasive percutaneous surgery [17] & 21 & 20 \\
\hline
\end{tabular}


9 articles included in this study. RevMan5.3 was employed to output the risk of bias chart (Figures 2,3). Nine articles were included in this meta-analysis (selection bias). There

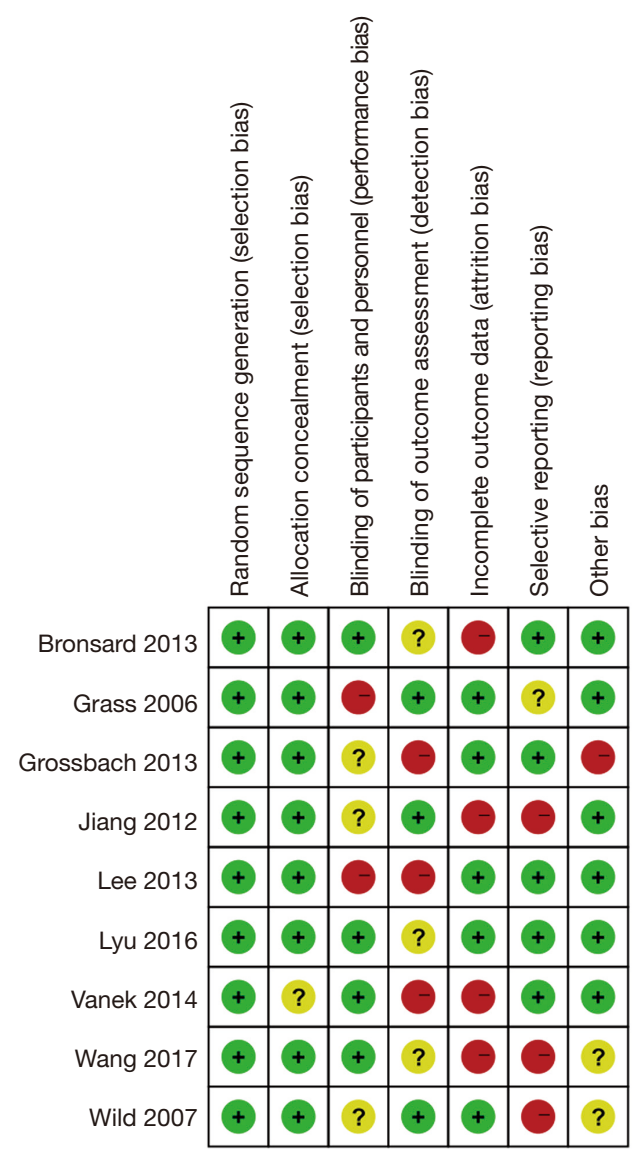

Figure 2 The bias-risk assessment diagram of the included articles. is 1 article of the allocation concealment (selection bias) for "unclear risk", and the other is "low risk". The evaluation of literature quality is implemented using the Cochrane Reviewer' Handbook. Then, the Jadad scale was adopted, and the evaluation found that the Jadad scale score included in the literature is greater than three points, so it is not necessary to perform sensitivity analysis.

\section{Operation time}

A total of 9 literatures met the requirements using percutaneous pedicle screw fixation and posterior percutaneous pedicle screw fixation. The results showed that the $95 \%$ CI was $-0.73(-0.94,-0.51), Z=6.71$, and $\mathrm{I}^{2}=73 \%$. Thus, heterogeneity was indicated $(\mathrm{P}<0.0001$; $\left.\mathrm{I}^{2}>50 \%\right)$, and the random effects model was selected. The analysis showed that percutaneous pedicle screw fixation and posterior percutaneous pedicle screw fixation were associated with a shorter surgical duration and a reduced incidence of intraoperative adverse reactions compared with open pedicle screw fixation. The funnel plot was basically symmetrical, and most of the data was on both sides of the central axis, indicating a low publication bias. Therefore, it is concluded that other procedures are more effective than internal fixation with open pedicle screws (Figures 4,5).

\section{Bleeding volume comparison}

A total of 8 articles met the requirements using percutaneous pedicle screw fixation and posterior percutaneous pedicle screw fixation. The results showed that the $95 \%$ CI was $-192.16(-213.23,-171.09), Z=17.87$,

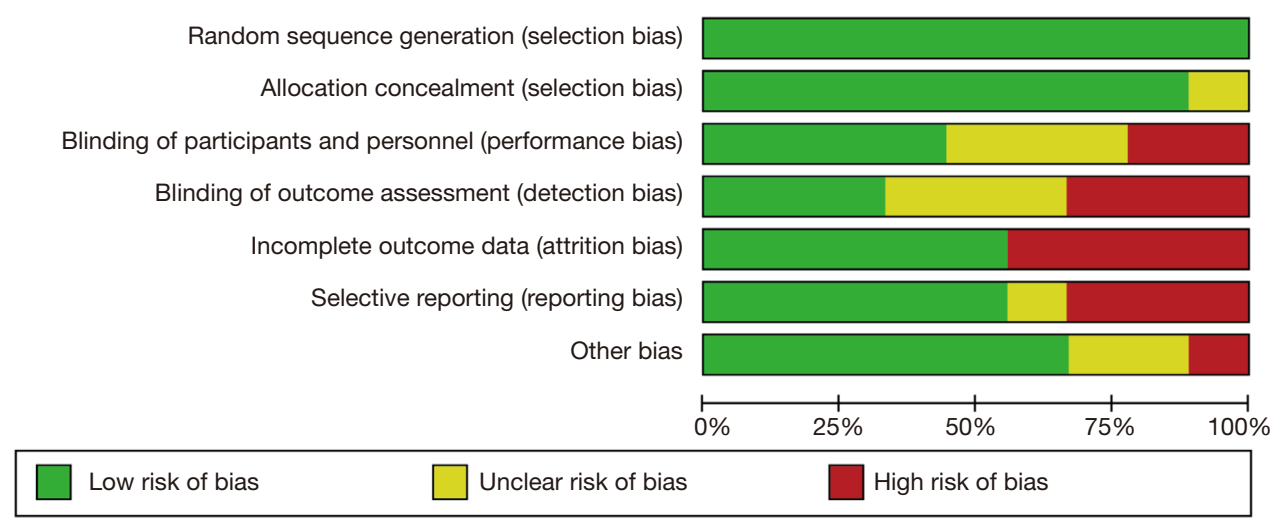

Figure 3 The bias evaluation bar graph of the included articles. 


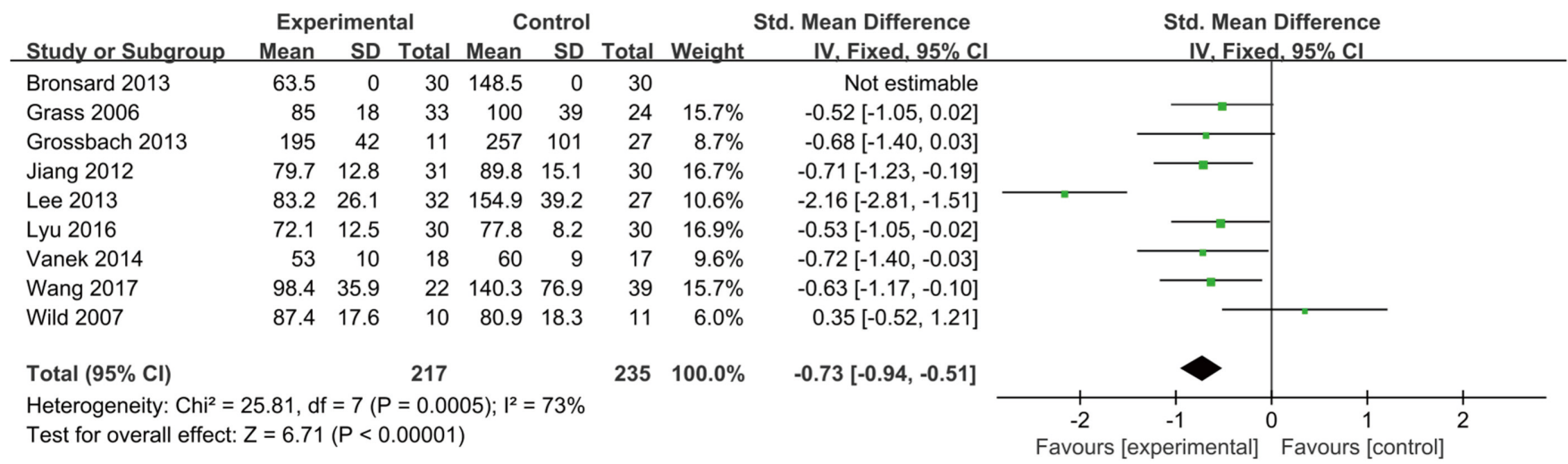

Figure 4 Forest plot for the comparison of other procedures and open pedicle screw internal fixation. SD, standard deviation; CI, confidence interval.

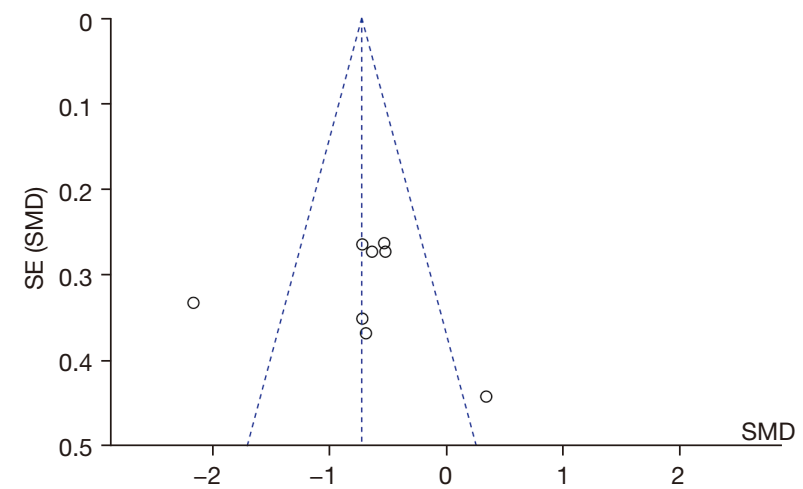

Figure 5 Funnel plot for the comparison of other procedures and open pedicle screw internal fixation. SE, standard error; SMD, standardized mean difference.

and $\mathrm{I}^{2}=99 \%$. Since $\mathrm{I}^{2}=99 \%>50 \%$, heterogeneity was indicated, so the random effects model was selected. It was concluded that percutaneous pedicle screw fixation and posterior percutaneous pedicle screw fixation resulted in less blood loss and less intraoperative adverse reactions than open pedicle screw fixation. The funnel plot was basically symmetrical, and most of the data was on both sides of the central axis, indicating a low publication bias. Therefore, the alternative method has less blood loss than the open pedicle screw fixation and prevents patients from developing intraoperative ischemic complications (Figures 6,7).

\section{Comparison of postoperative pain}

A total of 5 articles met the requirements using percutaneous pedicle screw fixation and posterior percutaneous pedicle screw fixation. The results showed that the $95 \%$ CI was -0.70 (-0.82, -0.57$), \mathrm{Z}=10.95$, and $\mathrm{I}^{2}=81 \%$. Since $\mathrm{I}^{2}=81 \%>50 \%$, there was heterogeneity, so the random effects model was selected. It was concluded that percutaneous pedicle screw fixation and posterior percutaneous pedicle screw fixation resulted in less pain than open pedicle screw fixation. The funnel plot was essentially symmetrical and most data were on both sides of the central axis, indicating a low publication bias. In summary, other procedures are less painful and lead to better patient compliance than open pedicle screw fixation (Figures 8,9).

\section{Postoperative screw dislocation rate}

A total of seven literatures met the requirements using percutaneous pedicle screw fixation and posterior percutaneous pedicle screw fixation. The results showed that the $95 \% \mathrm{CI}$ was $1.49(0.47,4.79), \mathrm{Z}=0.67$, and $\mathrm{I}^{2}=0 \%$. Since $\mathrm{I}^{2}=0 \%<50 \%$, there was no heterogeneity, so the fixed effects model was selected. It was concluded that percutaneous pedicle screw fixation and posterior percutaneous pedicle screw fixation had lower postoperative screw dislocation rates than open pedicle screw fixation. The funnel plot was essentially symmetrical and most data were on both sides of the central axis, indicating a low publication bias. In summary, other methods have a lower postoperative screw dislocation rate and fewer postoperative re-operations than open pedicle screw fixation (Figures 10,11).

\section{Comparison of postoperative infection rates}

A total of eight literatures met the requirements using 


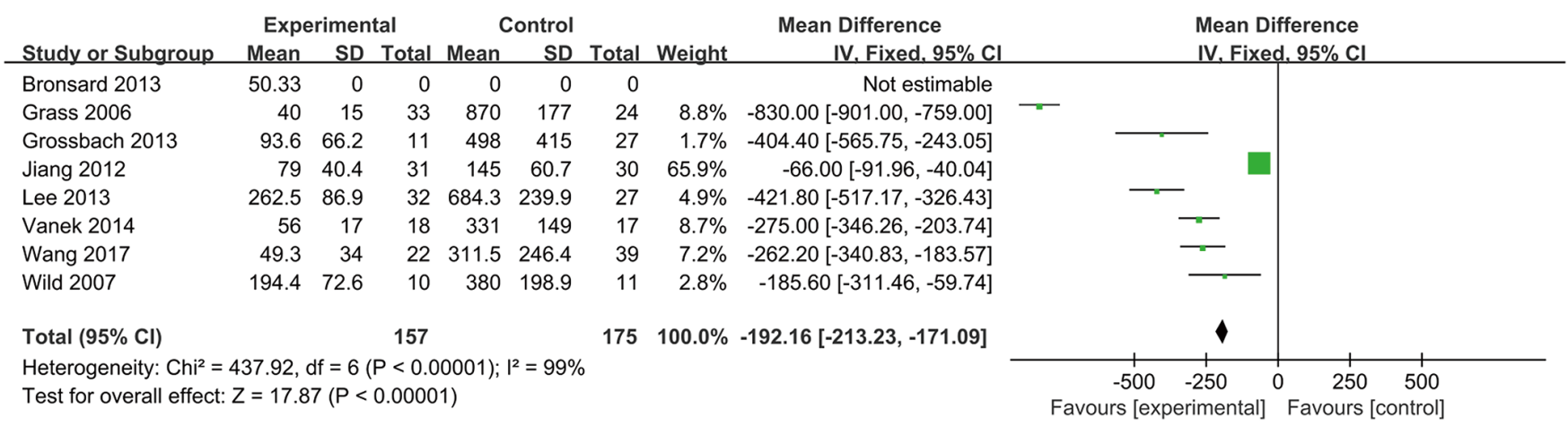

Figure 6 Forest plot for the comparison of bleeding volume between other procedures and open pedicle screw fixation. SD, standard deviation; CI, confidence interval.

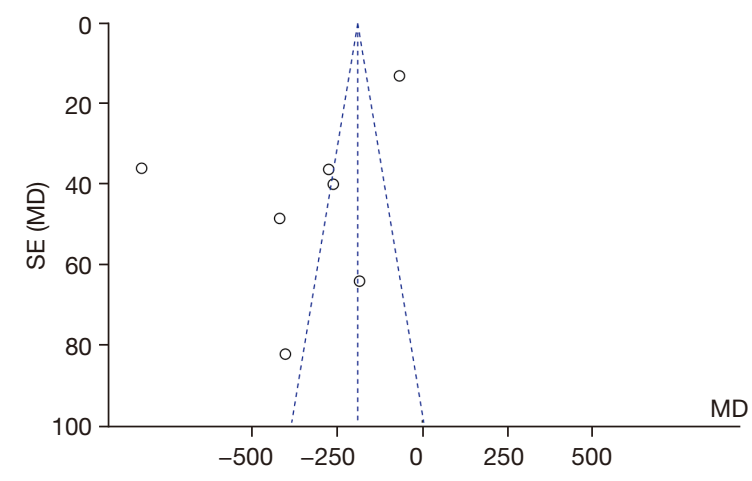

Figure 7 Funnel plot for the comparison of bleeding volume between other procedures and open pedicle screw fixation. SE, standard error; MD, mean difference.

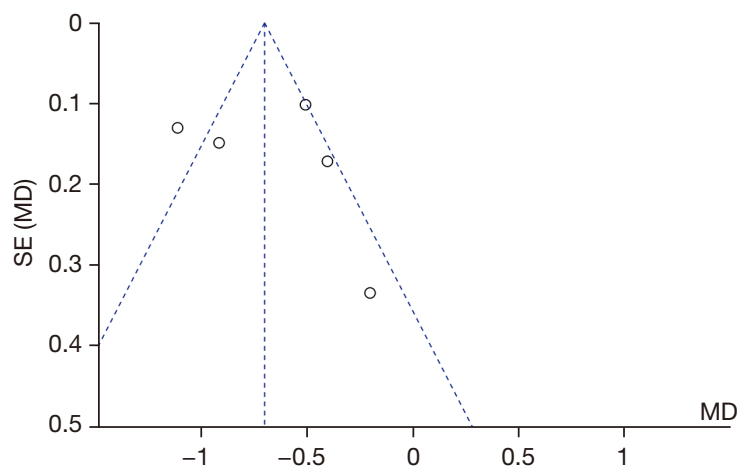

Figure 9 Funnel plot of pain perception comparison between other procedures and internal fixation with open pedicle screws. SE, standard error; MD, mean difference.

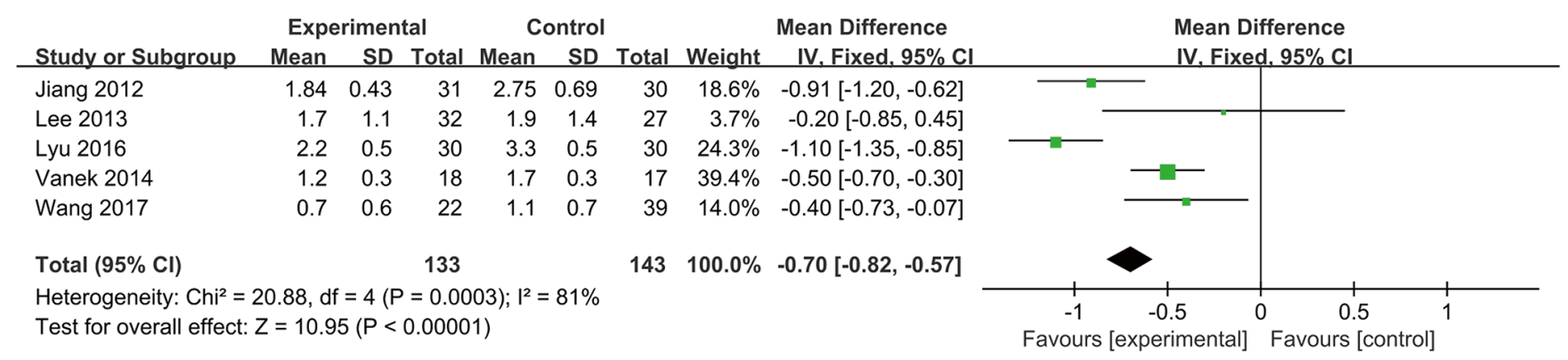

Figure 8 Forest plot of pain perception comparison between other procedures and internal fixation with open pedicle screws. SD, standard deviation; CI, confidence interval.

percutaneous pedicle screw fixation and posterior percutaneous pedicle screw fixation. The results showed that the $95 \%$ CI was $0.32(0.10,0.99), Z=1.97$, and
$\mathrm{I}^{2}=0 \%$. Since $\mathrm{I}^{2}=0 \%<50 \%$, there was no significant heterogeneity, so the fixed effects model is selected. It was concluded that percutaneous pedicle screw fixation 


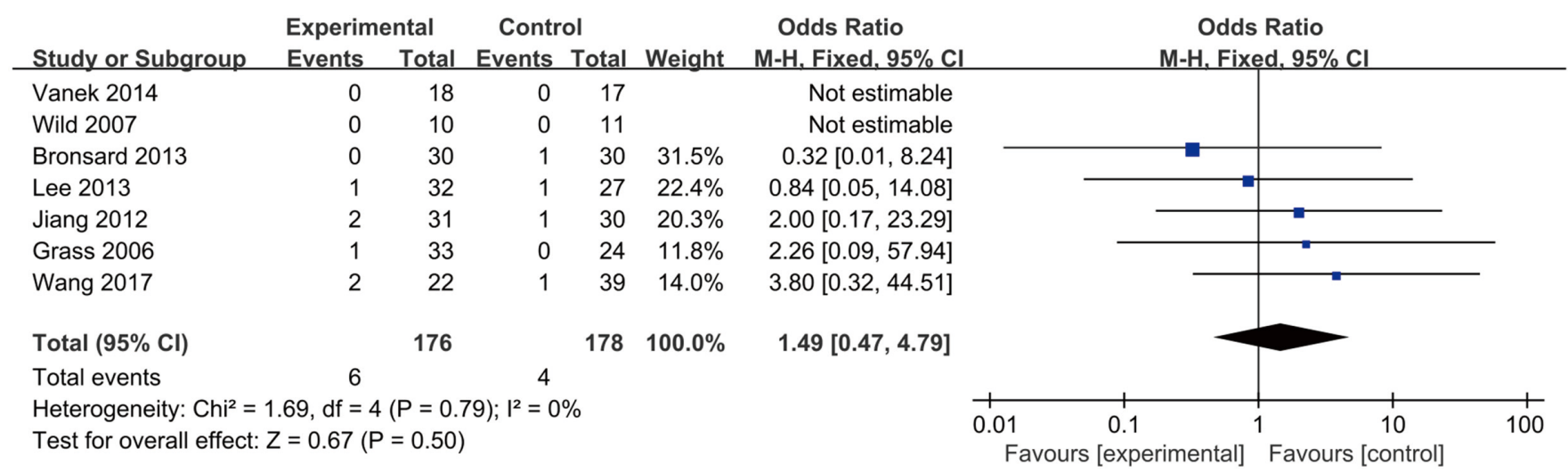

Figure 10 Forest plot of screw dislocations after other procedures and open pedicle screw fixation. CI, confidence interval.

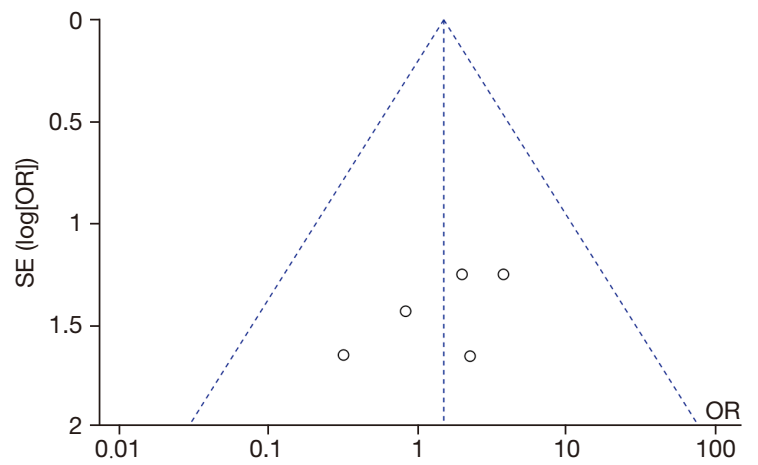

Figure 11 Funnel plot of screw dislocations after other procedures and open pedicle screw fixation. SE, standard error; OR, odds ratio.

and posterior percutaneous pedicle screw fixation had a lower postoperative infection rate than open pedicle screw fixation. The funnel plot was essentially symmetrical and most data were on both sides of the central axis, indicating a low publication bias. In summary, the infection rate and adverse reactions of the other methods are lower than those of the open pedicle screw fixation (Figures 12,13).

\section{Comparison of hospital stay}

A total of 4 literatures met the requirements using percutaneous pedicle screw fixation and posterior percutaneous pedicle screw fixation. The results showed that the $95 \% \mathrm{CI}$ was $-1.26(-1.82,-0.71), \mathrm{Z}=4.46$, and $\mathrm{I}^{2}=32 \%$. Since $\mathrm{I}^{2}=32 \%<50 \%$, there is heterogeneity, so the fixed effects model was selected. It was concluded that percutaneous pedicle screw fixation and posterior percutaneous pedicle screw fixation were associated with lower hospital stay compared to open pedicle screw fixation. The funnel plot was essentially symmetrical and mostly located in the $95 \%$ CI, indicating a low publication bias. Therefore, the other methods can reduce the hospital stay and better relieve the economic pressure of patients compared to open pedicle screw internal fixation (Figures 14,15).

\section{Discussion}

With the rapid development of medical technology, surgical treatment technology has become increasingly sophisticated. In terms of surgical treatment, more efficient surgical methods with less surgical trauma and shorter treatment time have been sought to replace the increasingly largescale traumatic surgery and traditional surgical methods that may result in multiple complications (21). The surgery should be determined according to the patient's fracture type, and there is a synthetic factor such as neurological dysfunction, damaged spine stability, and the level of clinical operation skills levels. Since different surgical methods have their own advantages and disadvantages, it is necessary to sufficiently combine the overall situation of the patient when the surgical method is selected. In recent years, the rapid development of minimally invasive surgery is attributable to the research and development of surgical instruments and new materials, resulting in less trauma and strong patient compliance, which is also beneficial to postoperative recovery. The shortened surgical time and hospital stay provides convenience to doctors, and more importantly, reduces the economic burden of patients (22). 


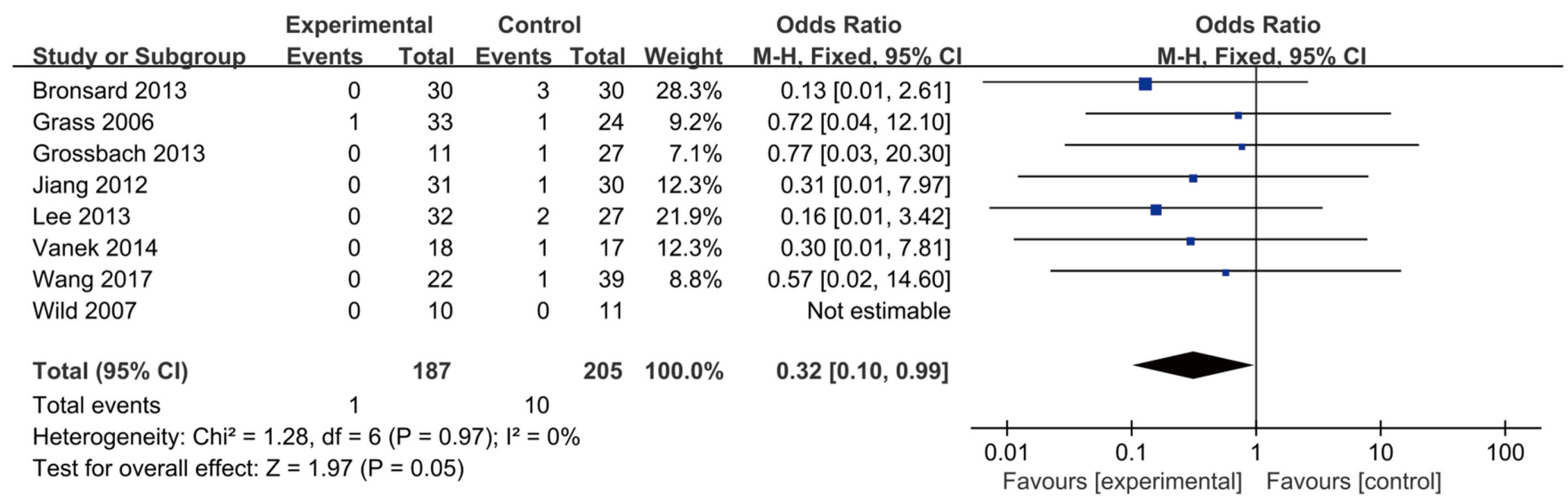

Figure 12 Forest plot of infection rates after other procedures and internal fixation with open pedicle screws. CI, confidence interval.

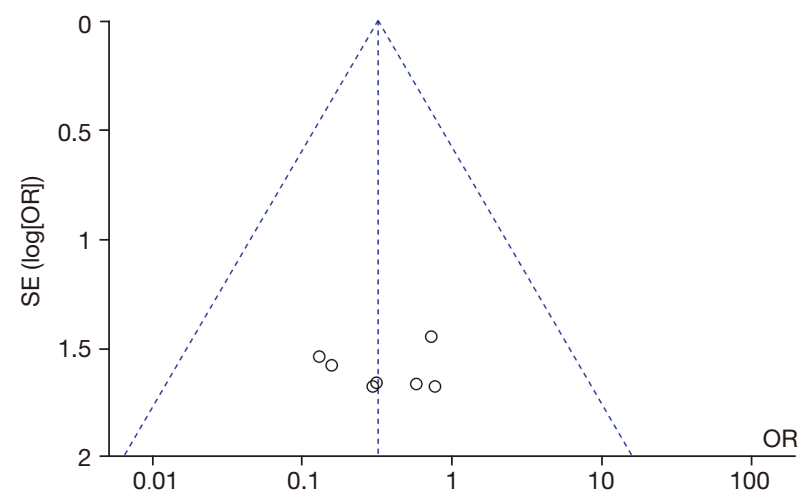

Figure 13 Funnel plot of infection rates after other procedures and internal fixation with open pedicle screws. SE, standard error; OR, odds ratio.

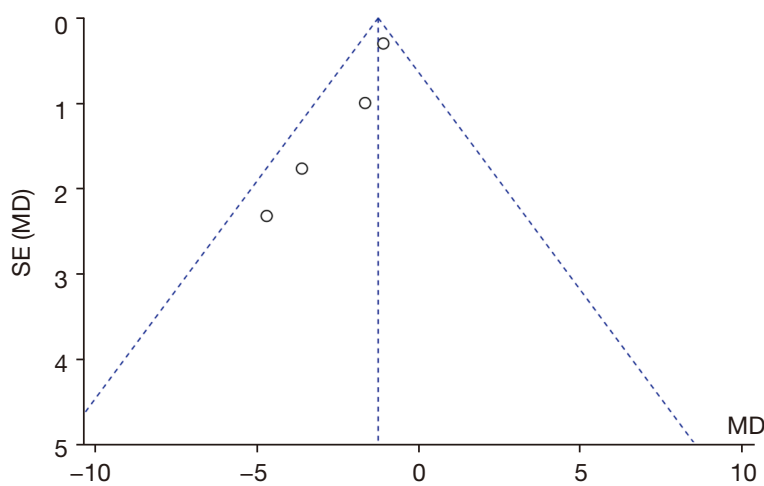

Figure 15 Funnel plot of hospital stay after other procedures and internal fixation with open pedicle screws. SE, standard error; MD, mean difference.

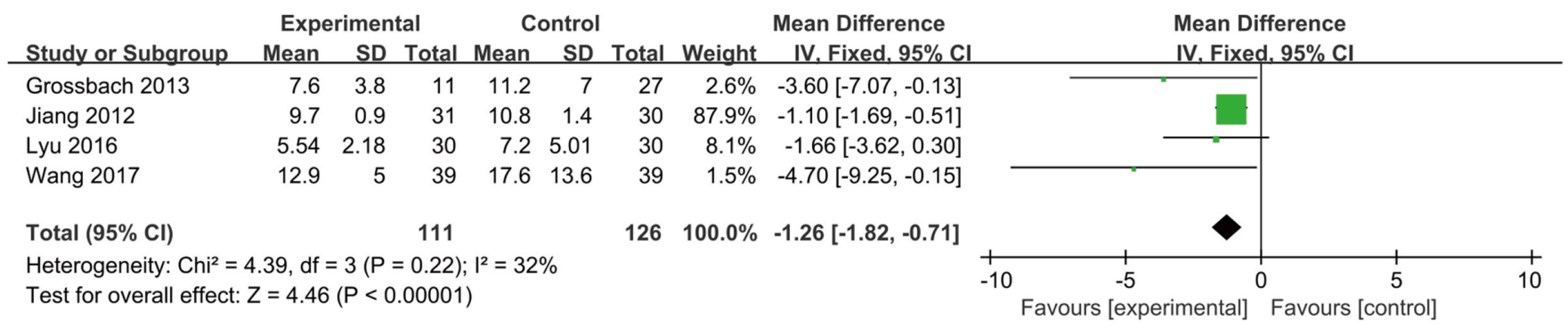

Figure 14 Forest plot of hospital stay after other procedures and internal fixation with open pedicle screws. CI, confidence interval.

Fractures of the thoracic spine are characterized by rapid onset and state change, as well as severe trauma. It can result in serious consequences such as pain, spinal instability, spinal deformation, and loss of function (23). If timely and comprehensive clinical treatment is not provided, there may be serious sequelae. Surgery is the most common treatment for thoracic fractures. Surgical vertebral fixation results in good contraction of the fracture and provides initial 
stabilization of the fracture, allowing the patient to get up and out of bed. As a result, the incidence of complications caused by a prolonged stay in bed is reduced. The main objectives of surgical treatment of thoracic fractures are effective decompression of the spinal tube, correction of spinal deformation, and reconstruction of spinal stability.

In many cases, clinical pedicle screw surgery requires careful dissection of the muscles surrounding the joint in search of fixation (24). The medial branch of the posterior branch of the spinal nerve passes through the root of the lateral process of the lower vertebral body and is not easily damaged. The lateral branch of the posterior ramus of the spinal nerve travels downward and outwards, innervating the deep muscles of the back along the way. However, due to the exposure requirements of the operative field in open surgery, a lamina spinner is required to prop up the muscularis. This process is prone to injury due to the high tension pulling of the posterior ramus of the spinal nerve.

From the included literature, patients who received other surgical methods had less blood loss and reduced pain compared with open surgery, which will become the development direction of spinal orthopedics. However, there are some disadvantages. Firstly, regarding the safety of percutaneous minimally invasive surgery, there is more $\mathrm{X}$-ray fluoroscopy, and thus, the amount of radiation for patients and doctors is increased, adversely affecting their health. The advent of computer-aided surgical navigation systems has helped in significantly reducing radiation exposure, but many hospitals do not yet have these systems due to their high costs. Takase (25) found that the mean radiation exposure time was longer than in the traditional open surgery group, and the effective dose in the transcutaneous group was more than three times that in the open group. Increasing the amount of radiation is a hazard to patients and a significant potential harm to surgeons who perform many surgeries over the years. Spinal surgeons, especially those who perform minimally invasive percutaneous surgery, receive higher radiation dose levels than the average orthopedic surgeon.

Secondly, it is difficult to re-enter the internal fixation device of percutaneous and posterior percutaneous fixation through the initial incision. The nail and rod system are often surrounded by tough scar tissue, and the fixation device is large, so repeated removal from the incision may cause secondary injuries. After the wound heals, the patient needs to re-open the surgical wound to insert the fixation device (26). Limited surgical experience and clinical skills may lead to damage or even fracture of the pedicle. The results of this meta-analysis indicate that, compared with traditional open surgery, other surgical methods have better surgical effect, less pain, and shorter surgical time. Percutaneous pedicle screw surgery requires a surgical incision of approximately $1 \mathrm{~cm}$ at each screw placement point. This incision is used to insert the needle, guide wire, and dilator tube. The screw is finally inserted with the help of the guide wire. The dilator tube is used for blunt separation of soft tissue and paravertebral muscle in the lower back, avoiding extensive dissection and electrosurgical burning in open surgery, and is thus very helpful in reducing postoperative pain, minimizing intraoperative bleeding, and improving patient recovery. The amount of bleeding is closely related to the type of spinal injury, such as a burst vertebral fracture, and bleeding has been an open question in these patients. Moreover, reducing the amount of blood loss during and after surgery is also conducive to postoperative recovery in high-risk elderly patients. Thus, it is possible to appropriately expand the indications for surgical treatment in this subgroup of patients. The reduction in surgical trauma results in a lower incidence of surgery-related complications, which can also promote early spinal stabilization, early recovery of mobility, and shorter hospital stays.

There are some limitations in this work that should be noted. Firstly, the included literatures in this meta-analysis are open literatures, and unpublished grey literatures were not included. Therefore, the results obtained from this bias may produce errors. Secondly, due to the relatively small number of cases included in this study, large sample size, multi-center, randomized controlled studies are still needed to confirm the reliability of the results of this metaanalysis. Thirdly, some postoperative efficacy indicators were not included [such as postoperative vertebral height, Oswestry disability index (ODI) score, and postoperative clinical satisfaction of patients], resulting in incomplete data analysis.

\section{Conclusions}

A total of 9 literatures were included in this meta-analysis. Compared with open surgery, the adoption of percutaneous pedicle screw and pedicle screw fixation had less blood loss, shorter operation time, shorter hospital stay, less pain, as well as lower screw dislocation and postoperative infection rates, indicating that the combination of percutaneous pedicle screw and pedicle screw fixation is more effective than open surgery. 


\section{Acknowledgments}

Funding: None.

\section{Footnote}

Reporting Checklist: The authors have completed the PRISMA reporting checklist. Available at https://dx.doi. org/10.21037/apm-21-3533

Conflicts of Interest: All authors have completed the ICMJE uniform disclosure form (available at https://dx.doi. org/10.21037/apm-21-3533). The authors have no conflicts of interest to declare.

Ethical Statement: The authors are accountable for all aspects of the work in ensuring that questions related to the accuracy or integrity of any part of the work are appropriately investigated and resolved.

Open Access Statement: This is an Open Access article distributed in accordance with the Creative Commons Attribution-NonCommercial-NoDerivs 4.0 International License (CC BY-NC-ND 4.0), which permits the noncommercial replication and distribution of the article with the strict proviso that no changes or edits are made and the original work is properly cited (including links to both the formal publication through the relevant DOI and the license). See: https://creativecommons.org/licenses/by-nc-nd/4.0/.

\section{References}

1. Kendler DL, Bauer DC, Davison KS, et al. Vertebral Fractures: Clinical Importance and Management. Am J Med 2016;129:221.e1-10.

2. Schousboe JT. Epidemiology of Vertebral Fractures. J Clin Densitom 2016;19:8-22.

3. Lentle B, Koromani F, Brown JP, et al. The Radiology of Osteoporotic Vertebral Fractures Revisited. J Bone Miner Res 2019;34:409-18.

4. Denis F. The three column spine and its significance in the classification of acute thoracolumbar spinal injuries. Spine (Phila Pa 1976) 1983;8:817-31.

5. Ferguson RL, Allen BL Jr. A mechanistic classification of thoracolumbar spine fractures. Clin Orthop Relat Res 1984;(189):77-88.

6. Roy-Camille R, Berteaux D, Saillant G. Unstable fractures of the spine. Surgical methods. Synthesis of the injured dorso-lumbar spine by plates screwed into vertebral pedicles. Orthop Traumatol Surg Res 2014;100:23-5.

7. Kocis J, Kelbl M, Kocis T, et al. Percutaneous versus open pedicle screw fixation for treatment of type A thoracolumbar fractures. Eur J Trauma Emerg Surg 2020;46:147-52.

8. Keorochana G, Pairuchvej S, Trathitephun W, et al. Comparative Outcomes of Cortical Screw Trajectory Fixation and Pedicle Screw Fixation in Lumbar Spinal Fusion: Systematic Review and Meta-analysis. World Neurosurg 2017;102:340-9.

9. Yang $M$, Zhao Q, Hao D, et al. Comparison of clinical results between novel percutaneous pedicle screw and traditional open pedicle screw fixation for thoracolumbar fractures without neurological deficit. Int Orthop 2019;43:1749-54.

10. Coric D, Rossi VJ, Peloza J, et al. Percutaneous, Navigated Minimally Invasive Posterior Cervical Pedicle Screw Fixation. Int J Spine Surg 2020;14:S14-21.

11. Zhou ZZ, Wang YM, Liang X, et al. Minimally Invasive Pedicle Screw Fixation Combined with Percutaneous Kyphoplasty Under O-Arm Navigation for the Treatment of Metastatic Spinal Tumors with Posterior Wall Destruction. Orthop Surg 2020;12:1131-9.

12. Bronsard N, Boli T, Challali M, et al. Comparison between percutaneous and traditional fixation of lumbar spine fracture: intraoperative radiation exposure levels and outcomes. Orthop Traumatol Surg Res 2013;99:162-8.

13. Grass R, Biewener A, Dickopf A, et al. Percutaneous dorsal versus open instrumentation for fractures of the thoracolumbar border. A comparative, prospective study. Unfallchirurg 2006;109:297-305.

14. Grossbach AJ, Dahdaleh NS, Abel TJ, et al. Flexiondistraction injuries of the thoracolumbar spine: open fusion versus percutaneous pedicle screw fixation. Neurosurg Focus 2013;35:E2.

15. Jiang XZ, Tian W, Liu B, et al. Comparison of a paraspinal approach with a percutaneous approach in the treatment of thoracolumbar burst fractures with posterior ligamentous complex injury: a prospective randomized controlled trial. J Int Med Res 2012;40:1343-56.

16. Lee JK, Jang JW, Kim TW, et al. Percutaneous shortsegment pedicle screw placement without fusion in the treatment of thoracolumbar burst fractures: is it effective?: comparative study with open short-segment pedicle screw fixation with posterolateral fusion. Acta Neurochir (Wien) 2013;155:2305-12; discussion 2312.

17. Lyu J, Chen K, Tang Z, et al. A comparison of three 
different surgical procedures in the treatment of type A thoracolumbar fractures: a randomized controlled trial. Int Orthop 2016;40:1233-8.

18. Vanek P, Bradac O, Konopkova R, et al. Treatment of thoracolumbar trauma by short-segment percutaneous transpedicular screw instrumentation: prospective comparative study with a minimum 2-year follow-up. J Neurosurg Spine 2014;20:150-6.

19. Wang H, Zhou Y, Li C, et al. Comparison of Open Versus Percutaneous Pedicle Screw Fixation Using the Sextant System in the Treatment of Traumatic Thoracolumbar Fractures. Clin Spine Surg 2017;30:E239-46.

20. Wild MH, Glees M, Plieschnegger C, et al. Five-year follow-up examination after purely minimally invasive posterior stabilization of thoracolumbar fractures: a comparison of minimally invasive percutaneously and conventionally open treated patients. Arch Orthop Trauma Surg 2007;127:335-43.

21. AlAsseri N, Swennen G. Minimally invasive orthognathic surgery: a systematic review. Int J Oral Maxillofac Surg 2018;47:1299-310.

22. Cloyd JM. Minimally Invasive Surgery for Palliation. Surg Oncol Clin N Am 2019;28:79-88.

23. Hershkovitz Y, Sheffer D, Peleg K, et al. Thoracic vertebrae fracture: Is it an indicator of abdominal injury? Am J Emerg Med 2021;43:235-7.

24. Xue X, Wang Y, Ji J, et al. Clinical application of cervical pedicle screw implantation technique under regional method. Zhongguo Xiu Fu Chong Jian Wai Ke Za Zhi 2020; 34:1515-20.

25. Takase K. Imaging Diagnosis and Interventional Radiology for Thoracic Surgery. Kyobu Geka 2018; 71:747-54.

26. Zhou XL, Liu DW, Mao YG, et al. Effect of tetrandine on gene expression of collagen type I, collagen type III and TGF-beta1 in scar tissue's of rabbits ear. Zhonghua Zheng Xing Wai Ke Za Zhi 2013; 29:406-12.

(English Language Editor: A. Kassem)
Cite this article as: Liang D, Deng X, Qian J, Han F, Zhou K. Comparison of different pedicle screw fixation schemes in the treatment of neurosurgical spinal fractures: systematic review and meta-analysis. Ann Palliat Med 2021;10(12):12678-12689. doi: 10.21037/apm-21-3533 